YGHP16-03

\title{
$Z_{B L}^{\prime}$ portal dark matter and LHC Run-2 results
}

\author{
Nobuchika Okada ${ }^{a}$ and Satomi Okada ${ }^{b}$ \\ ${ }^{a}$ Department of Physics and Astronomy, University of Alabama, Tuscaloosa, AL35487, USA \\ ${ }^{b}$ Graduate School of Science and Engineering, Yamagata University, \\ Yamagata 990-8560, Japan
}

\begin{abstract}
We consider a concise dark matter scenario in the minimal gauged $B-L$ extension of the Standard Model (SM), where the global $B-L$ (baryon number minus lepton number) symmetry in the SM is gauged, and three generations of right-handed neutrinos and a $B-L$ Higgs field are introduced. Associated with the $B-L$ gauge symmetry breaking by a VEV of the $B-L$ Higgs field, the seesaw mechanism for generating the neutrino mass is automatically implemented after the electroweak symmetry breaking in the SM. In this model context, we introduce a $Z_{2}$-parity and assign an odd parity for one right-handed neutrino while even parities for the other fields. Therefore, the dark matter candidate is identified as the right-handed Majorana neutrino with odd $Z_{2}$ parity, keeping the minimality of the particle content intact. When the dark matter particle communicates with the SM particles mainly through the $B-L$ gauge boson $\left(Z_{B L}^{\prime}\right.$ boson), its relic abundance is determined by only three free parameters, the $B-L$ gauge coupling $\left(\alpha_{B L}\right)$, the $Z_{B L}^{\prime}$ boson mass $\left(m_{Z^{\prime}}\right)$ and the dark matter mass $\left(m_{D M}\right)$. With the cosmological upper bound on the dark matter relic abundance we find a lower bound on $\alpha_{B L}$ as a function of $m_{Z^{\prime}}$. On the other hand, we interpret the recent LHC Run-2 results on search for $Z^{\prime}$ boson resonance to an upper bound on $\alpha_{B L}$ as a function of $m_{Z^{\prime}}$. Combining the two results we identify an allowed parameter region for this " $Z_{B L}^{\prime}$ portal" dark matter scenario, which turns out to be a narrow window with the lower mass bound of $m_{Z^{\prime}}>2.5 \mathrm{TeV}$.
\end{abstract}




\section{Introduction}

The neutrino mass matrix and the candidate of the dark matter particle are major missing pieces in the Standard Model (SM), that must be supplemented by the framework of beyond the SM. The seesaw mechanism [1] is probably the most natural way to incorporate the tiny neutrino masses and their flavor mixing, where right-handed neutrinos with Majorana masses are introduced. The minimal $B-L$ extended SM [2] is a very simple extension of the SM to naturally incorporate the seesaw mechanism. In this model, the accidental global $B-L$ (baryon number minus lepton number) symmetry in the SM is gauged, and an introduction of the three generations of right-handed neutrinos is required to keep the model from the gauge and gravitational anomalies. Associated with the $B-L$ gauge symmetry breaking, the right-handed neutrinos acquire Majorana masses, and the light neutrino Majorana masses are generated through the seesaw mechanism after the electroweak symmetry breaking. The mass spectrum of new particles introduced in the minimal $B-L$ model, the $B-L$ gauge boson $\left(Z_{B L}^{\prime}\right.$ boson), the right-handed Majorana neutrinos and the $B-L$ Higgs boson, is controlled by the $B-L$ symmetry breaking scale. If the breaking scale lies around the TeV scale, the $B-L$ model can be discovered at the Large Hadron Collider (LHC) in the near future.

One of the most promising candidates for the dark matter in the present universe is the Weakly Interacting Massive Particle (WIMP), which was in thermal equilibrium in the early universe and its relic density is determined by the interactions with the SM particles. It is a prime open question in particle physics and cosmology to identify the properties of the dark matter particle. There are, in general, various ways to supplement a dark matter particle to the SM. A simple and concise way to introduce a dark matter candidate in the context of the minimal $B-L$ model has been proposed in [3], where only a $Z_{2}$ parity is introduced without any extensions of the particle content of the model. An odd parity is assigned to one right-handed neutrino, while the other particles have even parties. As a result, the $Z_{2^{-}}$ odd right-handed neutrino plays a role of dark matter. The neutrino oscillation data can be reproduced by the so-called minimal seesaw [4] with two generations of the right-handed neutrinos, predicting one massless neutrino. Dark matter phenomenology in this model context has been investigated [3, 5]. The right-handed neutrino dark matter can annihilate into the SM particles through its interactions with the $Z_{B L}^{\prime}$ boson and two Higgs bosons which are realized as linear combinations of the SM Higgs and the $B-L$ Higgs bosons. Supersymmetric (SUSY) extension of the model has also been proposed [6], where the $B-L$ gauge symmetry is radiatively broken at the TeV scale through SUSY breaking effects [7, 8, 6]. In the SUSY extension of the model, the right-handed neutrino dark matter communicates with the SM

particles only through the $Z_{B L}^{\prime}$ boson, because SUSY forbids a mixing term between the SM 
Higgs and the $B-L$ Higgs fields in the superpotential at the renormalizable level.

Recently, the so-called $Z^{\prime}$ portal dark matter has a lot of attention [9]-[13], where a dark matter particle is introduced along with an extra gauge extension of the SM, and the dark matter particle communicates with the SM particles through the $Z^{\prime}$ gauge boson. The $Z^{\prime}$ boson as a mediator allows us to investigate a variety of dark matter physics, such as the dark matter relic abundance and the direct and indirect dark matter search. Interestingly, the search for $Z^{\prime}$ boson resonance at the LHC provides information that is complementary to dark matter physics. The minimal (SUSY) $B-L$ model with the right-handed neutrino dark matter discussed above is a very simple example of the $Z^{\prime}$ portal dark matter model, that we investigate in this papen 1 . Because of the simplicity of the model, dark matter physics is controlled by only three free parameters, the $B-L$ gauge coupling $\left(\alpha_{B L}\right)$, the $Z_{B L}^{\prime}$ boson mass $\left(m_{Z^{\prime}}\right)$ and the dark matter mass $\left(m_{D M}\right)$. We will identify allowed parameter regions of the model by considering the cosmological bound on the dark matter relic abundance and the most recent results by the LHC Run-2 on search for $Z^{\prime}$ boson resonance with dilepton final states [14, 15].

This paper is organized as follows. In the next section, we define the minimal $B-L$ model with the right-handed neutrino dark matter. In Sec. 3, we analyze the relic abundance of the $Z_{B L}^{\prime}$ portal dark matter, and identify a model parameter region to satisfy the upper bound on the dark matter relic abundance. In Sec. 4, we employ the results by the ATLAS and the CMS collaborations at the LHC Run-2 on search for the $Z^{\prime}$ boson resonance, and constrain the model parameter region. We find that the two parameter regions are complementary to each other, and lead to a narrow allowed window in the $\left(m_{Z^{\prime}}, \alpha_{B L}\right)$-plane. The last section is devoted to conclusions.

\section{The minimal $B-L$ model with a dark matter candidate}

We first define our model by the particle content listed on Table 1 . The global $B-L$ symmetry in the SM is gauged, and the three right-handed neutrinos $\left(N_{R}^{1}, N_{R}^{2}\right.$ and $\left.N_{R}\right)$ and a $B-L$ Higgs field $(\Phi)$ are introduced. The introduction of the $Z_{2}$ parity is crucial to incorporate a dark matter candidate in the model. Under this parity, the right-handed neutrino $N_{R}$ is assigned to be odd, while the other fields are even. The conservation of the $Z_{2}$ parity ensures the stability of the $Z_{2}$-odd $N_{R}$, and therefore, this right-handed neutrino is a unique dark matter candidate in the model [3].

\footnotetext{
1 As mentioned above, the right-handed neutrino dark matter can communicate with the SM particles also through the Higgs bosons and hence, it is a candidate of the so-called Higgs portal dark matter. See [3, 5] for detailed analysis on the $Z_{2}$-odd right-handed neutrino as the Higgs portal dark matter.
} 


\begin{tabular}{c|ccc|c|c} 
& $\mathrm{SU}(3)_{c}$ & $\mathrm{SU}(2)_{L}$ & $\mathrm{U}(1)_{Y}$ & $\mathrm{U}(1)_{B-L}$ & $Z_{2}$ \\
\hline$q_{L}^{i}$ & $\mathbf{3}$ & $\mathbf{2}$ & $1 / 6$ & $1 / 3$ & + \\
$u_{R}^{i}$ & $\mathbf{3}$ & $\mathbf{1}$ & $2 / 3$ & $1 / 3$ & + \\
$d_{R}^{i}$ & $\mathbf{3}$ & $\mathbf{1}$ & $-1 / 3$ & $1 / 3$ & + \\
\hline$\ell_{L}^{i}$ & $\mathbf{1}$ & $\mathbf{2}$ & $-\frac{1}{2}$ & -1 & + \\
$e_{R}^{i}$ & $\mathbf{1}$ & $\mathbf{1}$ & -1 & -1 & + \\
\hline$H$ & $\mathbf{1}$ & $\mathbf{2}$ & $-\frac{1}{2}$ & 0 & + \\
\hline$N_{R}^{j}$ & $\mathbf{1}$ & $\mathbf{1}$ & 0 & -1 & + \\
$N_{R}$ & $\mathbf{1}$ & $\mathbf{1}$ & 0 & -1 & - \\
$\Phi$ & $\mathbf{1}$ & $\mathbf{1}$ & 0 & +2 & + \\
\hline
\end{tabular}

Table 1: The particle content of the minimal $\mathrm{U}(1)_{B-L}$ extended SM with $Z_{2}$ parity. In addition to the SM particle content, the three right-handed neutrinos $N_{R}^{j}(j=1,2)$ and $N_{R}$ and a complex scalar $\Phi$ are introduced. The $Z_{2}$ parity is also introduced, under which the right-handed neutrino $N_{R}$ is odd, while the other fields are even.

The Yukawa sector of the SM is extended to have

$$
\mathcal{L}_{\text {Yukawa }} \supset-\sum_{i=1}^{3} \sum_{j=1}^{2} Y_{D}^{i j} \overline{\ell_{L}^{i}} H N_{R}^{j}-\frac{1}{2} \sum_{k=1}^{2} Y_{N}^{k} \Phi \overline{N_{R}^{k}{ }^{C}} N_{R}^{k}-\frac{1}{2} Y_{N} \Phi \overline{N_{R}^{C}} N_{R}+\text { h.c. }
$$

where the first term is the neutrino Dirac Yukawa coupling, and the second and third terms are the Majorana Yukawa couplings. Without loss of generality, the Majorana Yukawa couplings are already diagonalized in our basis. Note that because of the $Z_{2}$ parity only the two generation right-handed neutrinos are involved in the neutrino Dirac Yukawa coupling. Once the $B-L$ Higgs field $\Phi$ develops the vacuum expectation value (VEV), the $B-L$ gauge symmetry is broken and the Majorana mass terms for the right-handed neutrinos are generated. The seesaw mechanism [1] is automatically implemented in the model after the electroweak symmetry breaking. Because of the $Z_{2}$ parity, only two generation right-handed neutrinos are relevant to the seesaw mechanism, and this so-called minimal seesaw [4] possesses a number of free parameters $Y_{D}^{i j}$ and $Y_{N}^{k}$ enough to reproduce the neutrino oscillation data with a prediction of one massless eigenstate 2

The renormalizable scalar potential for the SM Higgs and the $B-L$ Higgs fields are generally given by

$$
V=m_{H}^{2}\left(H^{\dagger} H\right)+m_{\Phi}^{2}\left(\Phi^{\dagger} \Phi\right)+\lambda_{H}\left(H^{\dagger} H\right)^{2}+\lambda_{\Phi}\left(\Phi^{\dagger} \Phi\right)^{2}+\lambda_{H \Phi}\left(H^{\dagger} H\right)\left(\Phi^{\dagger} \Phi\right) .
$$

${ }^{2}$ When we consider leptogenesis scenario [16] in our model, only two right-handed neutrinos are involved. See, for example, [17] for detailed analysis of leptogenesis with two right-handed TeV scale neutrinos. The model can successfully generate a sufficient amount of baryon asymmetry in the universe. 
The parameters in the Higgs potential are suitably chosen for the Higgs fields to develop their VEVs as

$$
\langle H\rangle=\left(\begin{array}{c}
\frac{v}{\sqrt{2}} \\
0
\end{array}\right), \quad\langle\Phi\rangle=\frac{v_{B L}}{\sqrt{2}} .
$$

Associated with the $B-L$ symmetry breaking, the Majorana neutrinos $N_{R}^{j}(j=1,2)$, the dark matter particle $N_{R}$ and the $B-L$ gauge boson acquire their masses as

$$
m_{N}^{j}=\frac{Y_{N}^{j}}{\sqrt{2}} v_{B L}, \quad m_{D M}=\frac{Y_{N}}{\sqrt{2}} v_{B L}, \quad m_{Z^{\prime}}=2 g_{B L} v_{B L},
$$

where $g_{B L}$ is the $\mathrm{U}(1)_{B-L}$ gauge coupling.

The dark matter particle can communicate with the SM particles in two ways. One is through the Higgs bosons. In the Higgs potential of Eq. (2), the SM Higgs boson and the $B-L$ Higgs boson mix with each other in the mass eigenstates, and this Higgs boson mass eigenstates mediate the interactions between the dark matter particle and the SM particles. Dark matter physics with the interactions mediated by the Higgs bosons have been investigated in [3, 5]. The analysis involves 4 free parameters: Yukawa coupling $Y_{N}$ and 3 free parameters from the Higgs potential after two conditions of $v=246 \mathrm{GeV}$ and the SM-like Higgs boson mass fixed to be $125 \mathrm{GeV}$ are taken into account. The other way for the dark matter particle to communicate with the SM particles is through the $B-L$ gauge interaction with the $Z_{B L}^{\prime}$ boson. In this case, only three free parameters $\left(g_{B L}, m_{Z^{\prime}}\right.$ and $\left.m_{D M}\right)$ are involved in dark matter physics analysis. As we have stated in the previous section, we concentrate on dark matter physics mediated by the $Z_{B L}^{\prime}$ boson. When $\left|\lambda_{H \Phi}\right| \ll 1$, the Higgs bosons mediated interactions are negligibly small, and the dark matter particle communicates with the SM particles only through the $Z_{B L}^{\prime}$ boson. For example, this situation is realized in supersymmetric extension of our model [6], where $\lambda_{H \Phi}$ is forbidden by supersymmetry in the Higgs superpotential at the renormalizable level. When squarks and sleptons are all heavier than the dark matter particles, there is no essential difference in dark matter phenomenology between non-supersymmetric case

and supersymmetric case (see Ref. [6]). For a limited parameter choice, the $Z_{B L}^{\prime}$ portal dark matter scenario has been investigated in [5, 6].

\section{Cosmological constraint on $Z_{B L}^{\prime}$ portal dark matter}

The dark matter relic abundance is measured at the $68 \%$ limit as [18]

$$
\Omega_{D M} h^{2}=0.1198 \pm 0.0015
$$


In this section, we evaluate the relic abundance of the dark matter $N_{R}$ and identify an allowed parameter region that satisfies the upper bound on the dark matter relic density of $\Omega_{D M} h^{2} \leq$ 0.1213. The dark matter relic abundance is evaluated by integrating the Boltzmann equation given by

$$
\frac{d Y}{d x}=-\frac{s\left(m_{D M}\right)\langle\sigma v\rangle}{x^{2} H\left(m_{D M}\right)}\left(Y^{2}-Y_{E Q}^{2}\right)
$$

where temperature of the universe is normalized by the mass of the right-handed neutrino $x=m_{D M} / T, H\left(m_{D M}\right)$ is the Hubble parameter at $T=m_{D M}, Y$ is the yield (the ratio of the dark matter number density to the entropy density $s$ ) of the dark matter particle, $Y_{E Q}$ is the yield of the dark matter particle in thermal equilibrium, and $\langle\sigma v\rangle$ is the thermal average of the dark matter annihilation cross section times relative velocity. Explicit formulas of the quantities involved in the Boltzmann equation are as follows:

$$
\begin{aligned}
s\left(m_{D M}\right) & =\frac{2 \pi^{2}}{45} g_{\star} m_{D M}^{3}, \\
H\left(m_{D M}\right) & =\sqrt{\frac{4 \pi^{3}}{45} g_{\star}} \frac{m_{D M}^{2}}{M_{P l}}, \\
s Y_{E Q} & =\frac{g_{D M}}{2 \pi^{2}} \frac{m_{D M}^{3}}{x} K_{2}(x),
\end{aligned}
$$

where $M_{P l}=1.22 \times 10^{19} \mathrm{GeV}$ is the Planck mass, $g_{D M}=2$ is the number of degrees of freedom for the dark matter particle, $g_{\star}$ is the effective total number of degrees of freedom for particles in thermal equilibrium (in the following analysis, we use $g_{\star}=106.75$ for the SM particles), and $K_{2}$ is the modified Bessel function of the second kind. In our $Z_{B L}^{\prime}$ portal dark matter scenario, a pair of dark matter annihilates into the SM particles dominantly through the $Z_{B L}^{\prime}$ exchange in the $s$-channel. The thermal average of the annihilation cross section is given by

$$
\langle\sigma v\rangle=\left(s Y_{E Q}\right)^{-2} g_{D M}^{2} \frac{m_{D M}}{64 \pi^{4} x} \int_{4 m_{D M}^{2}}^{\infty} d s \hat{\sigma}(s) \sqrt{s} K_{1}\left(\frac{x \sqrt{s}}{m_{D M}}\right),
$$

where the reduced cross section is defined as $\hat{\sigma}(s)=2\left(s-4 m_{D M}^{2}\right) \sigma(s)$ with the total annihilation cross section $\sigma(s)$, and $K_{1}$ is the modified Bessel function of the first kind. The total cross section of the dark matter annihilation process $N N \rightarrow Z_{B L}^{\prime} \rightarrow f \bar{f}$ ( $f$ denotes the SM fermions) is calculated as

$$
\sigma(s)=\frac{\pi}{4} \alpha_{B L}^{2} \frac{\sqrt{s\left(s-4 m_{D M}^{2}\right)}}{\left(s-m_{Z^{\prime}}^{2}\right)^{2}+m_{Z^{\prime}}^{2} \Gamma_{Z^{\prime}}^{2}}\left[\frac{148}{9}+\frac{4}{3} \beta_{t}\left(1-\frac{1}{3} \beta_{t}^{2}\right)\right]
$$

with $\beta_{t}(s)=\sqrt{1-4 m_{t}^{2} / s}$, top quark mass of $m_{t}=173.34 \mathrm{GeV}$ and the total decay width of $Z_{B L}^{\prime}$ boson given by

$$
\Gamma_{Z^{\prime}}=\frac{\alpha_{B L}}{6} m_{Z^{\prime}}\left[\frac{37}{3}+\frac{1}{3} \beta_{t}\left(m_{Z^{\prime}}^{2}\right)\left(3-\beta_{t}\left(m_{Z^{\prime}}^{2}\right)^{2}\right)+\left(1-\frac{4 m_{D M}^{2}}{m_{Z^{\prime}}^{2}}\right)^{\frac{3}{2}} \theta\left(\frac{m_{Z^{\prime}}^{2}}{m_{D M}^{2}}-4\right)\right] .
$$




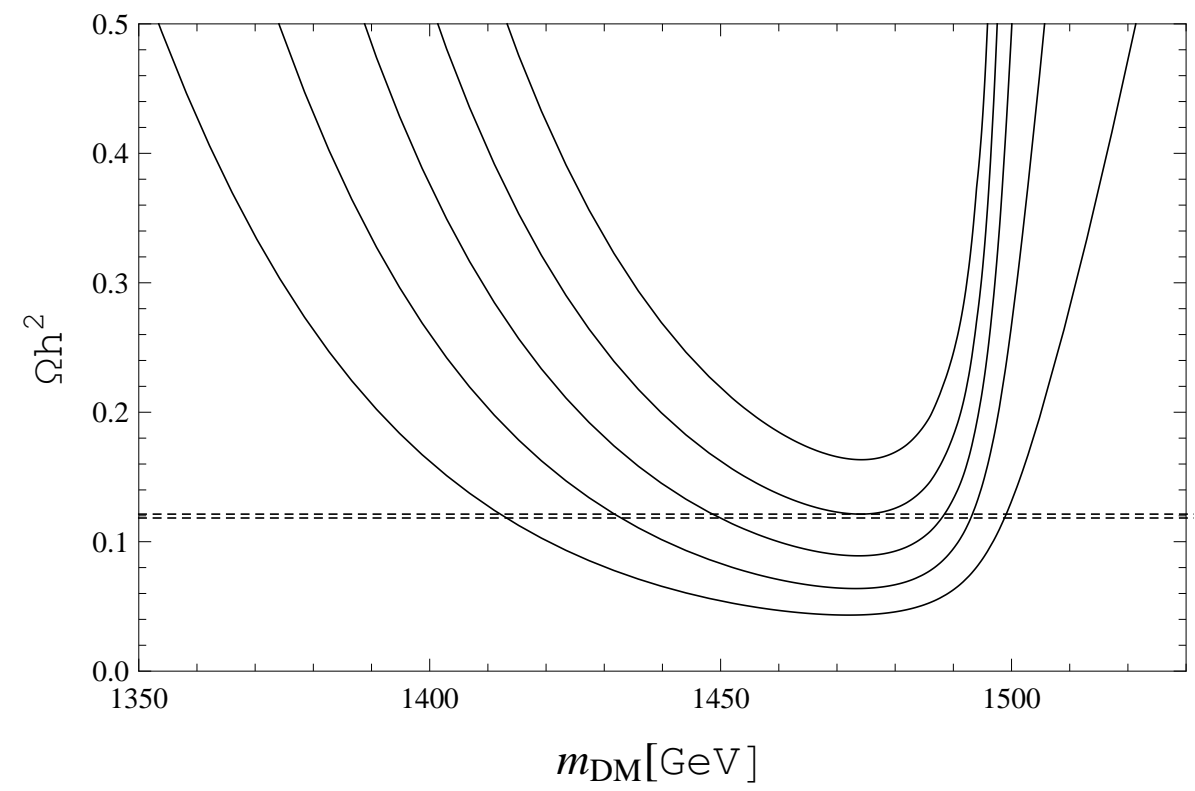

Figure 1: The relic abundance of the $Z_{B L}^{\prime}$ portal right-hard neutrino dark matter as a function of the dark matter mass $\left(m_{D M}\right)$ for $m_{Z^{\prime}}=3 \mathrm{TeV}$ and various values of the gauge coupling $\alpha_{B L}=0.001,0.0014,0.002,0.003$ and 0.005 (solid lines from top to bottom). The two horizontal lines denote the range of the observed dark matter relic density, $0.1183 \leq \Omega_{D M} h^{2} \leq 0.1213$.

Here, we have neglected all SM fermion masses except for $m_{t}$, and assumed $m_{N}^{j}>m_{Z^{\prime}} / 2$, for simplicity.

Now we solve the Boltzmann equation numerically, and find the asymptotic value of the yield $Y(\infty)$. Then, the dark matter relic density is evaluated as

$$
\Omega_{D M} h^{2}=\frac{m_{D M} s_{0} Y(\infty)}{\rho_{c} / h^{2}},
$$

where $s_{0}=2890 \mathrm{~cm}^{-3}$ is the entropy density of the present universe, and $\rho_{c} / h^{2}=1.05 \times$ $10^{-5} \mathrm{GeV} / \mathrm{cm}^{3}$ is the critical density. In our analysis, only three parameters, namely $\alpha_{B L}=$ $g_{B L}^{2} /(4 \pi), m_{Z^{\prime}}$ and $m_{D M}$, are involved. For $m_{Z^{\prime}}=3 \mathrm{TeV}$ and various values of the gauge coupling $\alpha_{B L}$, Fig. 1 shows the resultant dark matter relic abundance as a function of the dark matter mass $m_{D M}$, along with the observed bounds $0.1183 \leq \Omega_{D M} h^{2} \leq 0.1213$ [18] (two horizontal dashed lines). The solid lines from top to bottom correspond to the results for $\alpha_{B L}=0.001,0.0014,0.002,0.003$ and 0.005 , respectively. We can see that only if the dark matter mass is close to half of the $Z_{B L}^{\prime}$ boson mass, the observed relic abundance can be reproduced. In other words, normal values of the dark matter annihilation cross section leads to over-abundance, and it is necessary that an enhancement of the cross section through the $Z_{B L}^{\prime}$ boson resonance in the $s$-channel annihilation process. 

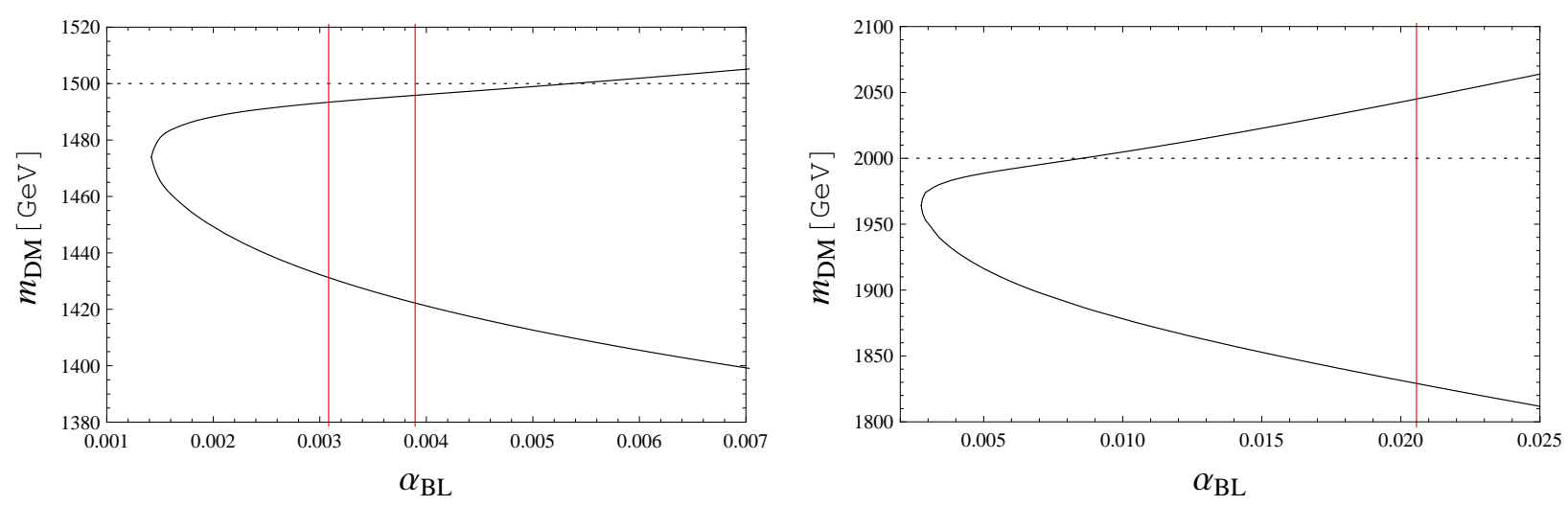

Figure 2: The dark matter mass as a function of $\alpha_{B L}$ for $m_{Z^{\prime}}=3 \mathrm{TeV}$ (left panel) and $m_{Z^{\prime}}=4 \mathrm{TeV}$ (right panel). Along the solid (black) curve in each panel, $\Omega_{D M} h^{2}=0.1198$ is satisfied. The dotted lines correspond to $m_{D M}=m_{Z^{\prime}} / 2$. The vertical solid lines (in red) denote the upper bound on $\alpha_{B L}$ obtained from the recent LHC Run-2 results (see Figs. 4 and 5 ). In the left panel, the left vertical line represents the constraint from the ATLAS result [14], while the right one is from the CMS result [15]. In the right panel, the vertical line represents the constraint from the ATLAS result [14].

For a fixed $m_{D M}$ in the Fig. 1, the resultant relic abundance becomes larger as the gauge coupling $\alpha_{B L}$ is lowered. As a result, there is a lower bound on $\alpha_{B L}$ in order to satisfy the cosmological upper bound on the dark matter relic abundance $\Omega_{D M} h^{2} \leq 0.1213$. For a $\alpha_{B L}$ value larger than the lower bound, we can find two values of $m_{D M}$ which result in the center value of the observed relic abundance $\Omega_{D M} h^{2}=0.1198$. In Fig. 2, we show the dark matter mass resulting $\Omega_{D M} h^{2}=0.1198$ as a function of $\alpha_{B L}$. The left panel shows the result for $m_{Z^{\prime}}=3 \mathrm{TeV}$, while the corresponding results for $m_{Z^{\prime}}=4 \mathrm{TeV}$ is shown in the right panel. As a reference, we also show the dotted lines corresponding to $m_{D M}=m_{Z^{\prime}} / 2$. In Fig. 1, we see that the minimum relic abundance is achieved by a dark matter mass which is very close to, but smaller than $m_{Z^{\prime}} / 2$. Although the annihilation cross section of Eq. (9) has a peak at $\sqrt{s}=m_{Z^{\prime}}$, the thermal averaged cross section given in Eq. (8) includes the integral of the product of the reduced cross section and the modified Bessel function $K_{1}$. Our results indicate that for $m_{D M}$ taken to be slightly smaller than $m_{Z^{\prime}} / 2$, the thermal averaged cross section is larger than the one for $m_{D}=m_{Z^{\prime}} / 2$.

As mentioned above, for a fixed $Z_{B L}^{\prime}$ boson mass, we can find a corresponding lower bound on the gauge coupling $\alpha_{B L}$ in order for the resultant relic abundance not to exceed the cosmological upper bound $\Omega_{D M} h^{2}=0.1213$. Figure 3 depicts the lower bound of $\alpha_{B L}$ as a function of $m_{Z^{\prime}}$ (solid (black) line). Along this solid (black) line, we find that the dark matter mass is approximately given by $m_{D M} \simeq 0.49 m_{Z^{\prime}}$. The dark matter relic abundance exceeds the 


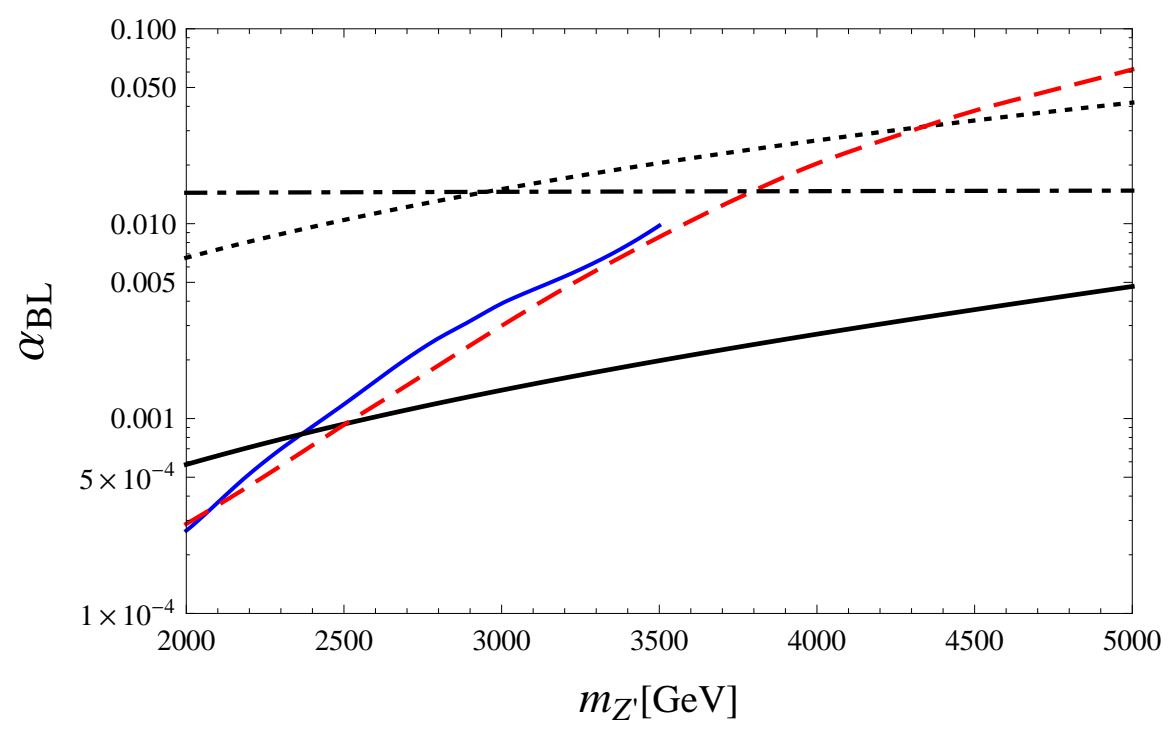

Figure 3: Allowed parameter region for the $Z_{B L}^{\prime}$ portal dark matter scenario. The solid (black) line shows the lower bound on $\alpha_{B L}$ as a function of $m_{Z^{\prime}}$ to satisfy the cosmological upper bound on the dark matter relic abundance. The dashed line (in red) shows the upper bound on $\alpha_{B L}$ as a function of $m_{Z^{\prime}}$ from the search results for $Z^{\prime}$ boson resonance by the ATLAS collaboration, while the diagonal line (in blue) in the range of $2000 \mathrm{GeV} \leq m_{Z^{\prime}} \leq 3500 \mathrm{GeV}$ denotes the upper bound obtained from the result by the CMS collaboration. The LEP bound is depicted as the dotted line. The regions above these dashed, (blue) solid and dotted lines are excluded. We also show a theoretical upper bound on $\alpha_{B L}$ to avoid the Landau pole of the running $B-L$ gauge coupling below the Planck mass $M_{P l}$.

cosmological upper bound in the region below the solid (black) line. Along with the other constraints that will be obtained in the next section, Fig. 3 is our main results in this paper.

\section{Interpretation of LHC Run-2 results}

Very recently, the LHC Run-2 started its operation with a $13 \mathrm{TeV}$ collider energy. Preliminary results from the ATLAS and the CMS collaborations have been reported [19]. The Run-2 results have provided constraints on new physics models, some of which are more severe than those by the LHC Run-1 results. The ATLAS and the CMS collaborations continue search for $Z^{\prime}$ boson resonance with dilepton final states at the LHC Run-2, and have improved the upper limits of the $Z^{\prime}$ boson production cross section from those in the LHC Run-1 [20, 21]. Employing the LHC Run-2 results, we will derive an upper bound on $\alpha_{B L}$ as a function of $m_{Z^{\prime}}$. Since we have obtained in the previous section the lower bound on $\alpha_{B L}$ as a function of $m_{Z^{\prime}}$ from the constraint on the dark matter relic abundance, the LHC Run-2 results are complementary to 

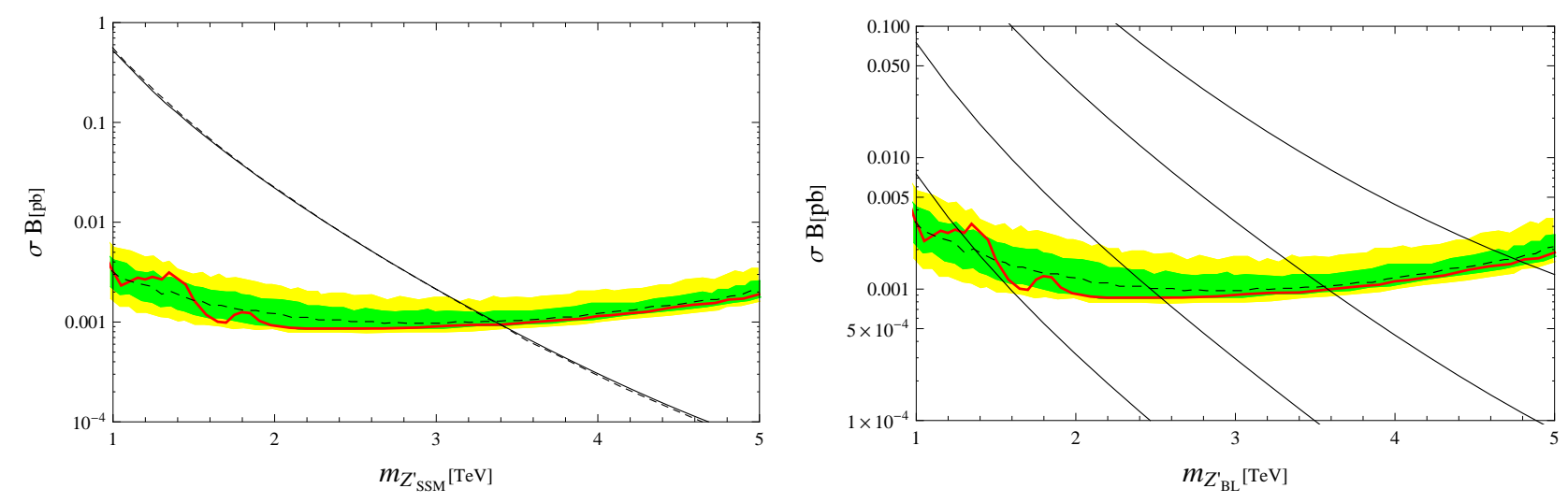

Figure 4: Left panel: the cross section as a function of the $Z_{S S M}^{\prime}$ mass (solid line) with $k=1.31$, along with the ATLAS result in Ref. [14] from the combined dielectron and dimuon channels. Right panel: the cross sections calculated for various values of $\alpha_{B L}$ with $k=1.31$. The solid lines from left to right correspond to $\alpha_{B L}=0.0001,0.001,0.01$ and 0.05 , respectively.

the cosmological constraint. As a result, the parameter space of the $Z_{B L}^{\prime}$ portal dark matter scenario is severally constrained once the two constraints are combined.

Let us calculate the cross section for the process $p p \rightarrow Z_{B L}^{\prime}+X \rightarrow \ell^{+} \ell^{-}+X$. The differential cross section with respect to the invariant mass $M_{\ell \ell}$ of the final state dilepton is described as

$$
\frac{d \sigma}{d M_{\ell \ell}}=\sum_{a, b} \int_{\frac{M_{\ell \ell}^{2}}{E_{\mathrm{CM}}^{2}}}^{1} d x \frac{2 M_{\ell \ell}}{x E_{\mathrm{CM}}^{2}} f_{a}\left(x, Q^{2}\right) f_{b}\left(\frac{M_{\ell \ell}^{2}}{x E_{\mathrm{CM}}^{2}}, Q^{2}\right) \hat{\sigma}\left(q \bar{q} \rightarrow Z_{B L}^{\prime} \rightarrow \ell^{+} \ell^{-}\right),
$$

where $f_{a}$ is the parton distribution function for a parton "a", and $E_{\mathrm{CM}}=13 \mathrm{TeV}$ is the centerof-mass energy of the LHC Run-2. In our numerical analysis, we employ CTEQ6L [22] for the parton distribution functions with the factorization scale $Q=m_{Z^{\prime}}$. Here, the cross section for the colliding partons is given by

$$
\hat{\sigma}=\frac{4 \pi}{81} \alpha_{B L}^{2} \frac{M_{\ell \ell}^{2}}{\left(M_{\ell \ell}^{2}-m_{Z^{\prime}}^{2}\right)^{2}+m_{Z^{\prime}}^{2} \Gamma_{Z^{\prime}}^{2}} .
$$

By integrating the differential cross section over a range of $M_{\ell \ell}$ set by the ATLAS and the CMS analysis, respectively, we obtain the cross section to be compared with the upper bounds obtained by the ATLAS and the CMS collaborations.

In the analysis by the ATLAS and the CMS collaborations, the so-called sequential SM $Z^{\prime}$ $\left(Z_{S S M}^{\prime}\right)$ model [23] has been considered as a reference model. We first analyze the sequential $Z^{\prime}$ model to check a consistency of our analysis with the one by the ATLAS collaboration. In the sequential $Z^{\prime}$ model, the $Z_{S S M}^{\prime}$ boson has exactly the same couplings with quarks and leptons as the SM $Z$ boson. With the couplings, we calculate the cross section of the process 

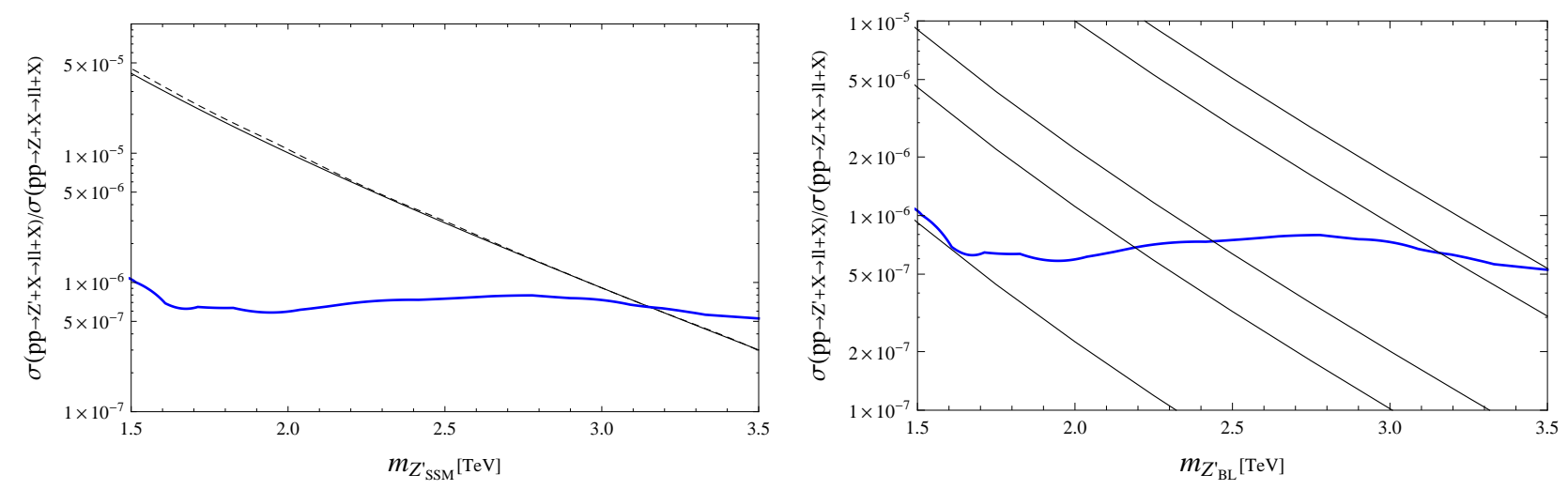

Figure 5: Left panel: the cross section ratio as a function of the $Z_{S S M}^{\prime}$ mass (solid line) with $k=1.80$, along with the CMS result in Ref. [15] from the combined dielectron and dimuon channels. Right panel: the cross section ratios calculated for various values of $\alpha_{B L}$ with $k=1.80$. The solid lines from left to right correspond to $\alpha_{B L}=0.0001,0.0005,0.001$, 0.005 and 0.01 , respectively.

$p p \rightarrow Z_{S S M}^{\prime}+X \rightarrow \ell^{+} \ell^{-}+X$ like Eq. (12). By integrating the differential cross section in the region of $128 \mathrm{GeV} \leq M_{\ell \ell} \leq 6000 \mathrm{GeV}$ [20], we obtain the cross section of the dilepton production process as a function of $Z_{S S M}^{\prime}$ boson mass 3 Our result is shown as a solid line in the left panel on Fig. 4, along with the plot presented by the ATLAS collaboration [14. In the analysis in the ATLAS paper, the lower limit of the $Z_{S S M}^{\prime}$ boson mass is found to be $3.4 \mathrm{TeV}$, which is read from the intersection point of the theory prediction (diagonal dashed line) and the experimental cross section bound (horizontal solid curve (in red)). In order to take into account the difference of the parton distribution functions used in the ATLAS and our analysis and QCD corrections of the process, we have scaled our resultant cross section by a factor $k=1.31$, with which we can obtain the same lower limit of the $Z_{S S M}^{\prime}$ boson mass as $3.4 \mathrm{TeV}$. We can see that our result with the factor of $k=1.31$ is very consistent with the theoretical prediction (diagonal dashed line) presented in Ref. [14]. This factor is used in our analysis of the $Z_{B L}^{\prime}$ production process. Now we calculate the cross section of the process $p p \rightarrow Z_{B L}^{\prime}+X \rightarrow \ell^{+} \ell^{-}+X$ for various values of $\alpha_{B L}$, and our results are shown in the right panel of Fig. 4, along with the plot in Ref. [14]. The diagonal solid lines from left to right correspond to $\alpha_{B L}=0.0001,0.001,0.01$ and 0.05, respectively. From the intersections of the horizontal curve and diagonal solid lines, we can read off a lower bound on the $Z_{B L}^{\prime}$ boson mass for a fixed $\alpha_{B L}$ value. In this way, we have obtained the upper bound on $\alpha_{B L}$ as a function of the $Z_{B L}^{\prime}$ boson mass, which is depicted in Fig. 3 (dashed (red) line).

\footnotetext{
${ }^{3}$ Since the decay width of the $Z_{S S M}^{\prime}$ boson is narrow, the cross section is almost determined by the integral in the vicinity of the resonance pole.
} 
We apply the same strategy and compare our result for the $Z_{S S M}^{\prime}$ model with the one by the CMS collaboration [15]. According to the CMS analysis, we integrate the differential cross section in the range of $0.97 m_{Z_{S S M}^{\prime}} \leq M_{\ell \ell} \leq 1.03 m_{Z_{S S M}^{\prime}}$. In the CMS analysis, a limit has been set on the ratio of the $Z_{S S M}^{\prime}$ boson cross section to the $Z / \gamma^{*}$ cross section in a mass window of 60 to $120 \mathrm{GeV}$, which is predicted to be $1928 \mathrm{pb}$. Our result is shown as a diagonal solid line in the left panel of Fig. 5, along with the plot presented in Ref. [15]. The analysis in this CMS paper leads to the lower limit of the $Z_{S S M}^{\prime}$ boson mass as $3.15 \mathrm{TeV}$, which is read from the intersection point of the theory prediction (diagonal dashed line) and the experimental cross section bound (horizontal solid curve (in blue)). In order to obtain the same lower mass limit of $m_{Z_{S S M}^{\prime}} \geq 3.15 \mathrm{TeV}$, we have introduced a factor $k=1.80$. The left panel shows that our results are very consistent with the theoretical cross section presented in Ref. [15].

With the factor of $k=1.80$, we calculate the cross section of the process $p p \rightarrow Z_{B L}^{\prime}+X \rightarrow$ $\ell^{+} \ell^{-}+X$ for various values of $\alpha_{B L}$, and our results are shown in the right panel of Fig. 5 , along with the plot in Ref. [15]. The diagonal solid lines from left to right correspond to $\alpha_{B L}=0.0001$, 0.0005, 0.001, 0.005 and 0.01, respectively. From the intersections of the horizontal (blue) curve and diagonal solid lines, we can read off a lower bound on the $Z_{B L}^{\prime}$ boson mass for a fixed $\alpha_{B L}$ value. In Fig. 3, the diagonal solid (blue) line in the range of $2000 \mathrm{GeV} \leq m_{Z_{B L}^{\prime}} \leq 3500 \mathrm{GeV}$ shows the upper bound on $\alpha_{B L}$ as a function of the $Z_{B L}^{\prime}$ boson mass. The ATLAS and the CMS bounds we have obtained are consistent with each other. The ATLAS bound is slightly more severe than the CMS bound, and applicable to a higher mass range up to $m_{Z^{\prime}}=5000$ $\mathrm{GeV}$.

In Fig. 3, we also show the LEP bound as the dotted line which is obtained from the search for effective 4-Fermi interactions mediated by the $Z_{B L}^{\prime}$ boson [24]. An updated limit with the final LEP 2 data [25] is found to be [26]

$$
\frac{m_{Z^{\prime}}}{g_{B L}} \geq 6.9 \mathrm{TeV}
$$

at $95 \%$ confidence level. We find that the ATLAS bound at the LHC Run-2 is more severe than the LEP bound for $m_{Z^{\prime}} \lesssim 4.3 \mathrm{TeV}$. In order to avoid the Landau pole of the running $B-L$ coupling $\alpha_{B L}(\mu)$ below the Plank mass, $1 / \alpha_{B L}\left(M_{P l}\right)>0$, we find

$$
\alpha_{B L}<\frac{\pi}{6 \ln \left[\frac{M_{P l}}{m_{Z^{\prime}}}\right]},
$$

which is shown as the dashed-dotted line in Fig. 3. Here, the gauge coupling $\alpha_{B L}$ used in our analysis for dark matter physics and LHC physics is defined as the running gauge coupling $\alpha_{B L}(\mu)$ at $\mu=m_{Z^{\prime}}$, and we have employed the renormalization group equation at the one-loop level with $m_{N}^{1}=m_{N}^{2}=m_{\Phi}=m_{Z^{\prime}}$, for simplicity. 


\section{Conclusions}

We have considered the minimal gauged $B-L$ extension of the Standard Model, which is free from all the gauge and gravitational anomalies and automatically incorporates the neutrino mass and flavor mixing through the seesaw mechanism. We have extended this model by introducing a $Z_{2}$ parity, so that a dark matter candidate is supplemented and identified as an $Z_{2}$-odd right-handed neutrino. No extension of the particle content from the one of the minimal $B-L$ model is needed. In this model, the dark matter particle communicates with the Standard Model particles through the $B-L$ gauge boson $\left(Z_{B L}^{\prime}\right.$ boson). Since the $B-L$ charges for all particles are fixed, physics of this " $Z_{B L}^{\prime}$ portal" dark matter scenario is controlled by only three parameters, namely, the gauge coupling, the $Z_{B L}^{\prime}$ boson mass, and the dark matter mass. Imposing the cosmological upper bound on the dark matter relic density, we have found the lower bound on the $B-L$ gauge coupling as a function of the $Z_{B L}^{\prime}$ boson mass. Search results for $Z^{\prime}$ boson resonance by the ATLAS and CMS collaborations at the LHC Run-2 provide the information that is complementary to the cosmological bound on the " $Z_{B L}^{\prime}$ portal" dark matter scenario. We have interpreted the $Z^{\prime}$ boson resonance search results at the LHC Run-2, and obtained the upper bound on the $B-L$ gauge coupling as a function of the $Z_{B L}^{\prime}$ boson mass. Similar upper bounds on the $B-L$ gauge coupling can be obtained through results by the LEP experiment of search for effective 4-Fermi interactions mediated by the $Z_{B L}^{\prime}$ boson and the requirement to maintain the running $B-L$ gauge coupling in perturbative regime up to the Planck mass. Putting all together, our final result is shown in Fig. 3. We have identified the allowed parameter region for the " $Z_{B L}^{\prime}$ portal" dark matter scenario, which turns out to be narrow and leads to the lower bound on the $Z_{B L}^{\prime}$ boson mass of $m_{Z^{\prime}}>2.5 \mathrm{TeV}$.

In the present model, the Standard Model fermions couple with the $Z_{B L}^{\prime}$ boson through the vector current, while the dark matter particle has the axial current coupling because of its Majorana nature. Hence, the elastic scattering cross section of the dark matter particle with nuclei vanishes in the non-relativistic limit, and the direct and indirect search for the dark matter particle is not applicable to the present scenario. Our model can be easily extended to have more general U(1) gauge symmetry [27], while keeping the same minimal particle content. In this case, the axial vector couplings between the Standard Model fermions with the $Z^{\prime}$ gauge boson arise in general, and the dark matter particle can scatter off nuclei. In the context of the sequential $Z^{\prime}$ model as a reference, the constraints from the direct and indirect dark matter search on the $Z^{\prime}$ portal dark matter scenario have been investigated in Ref. [12]. Several representative $Z^{\prime}$ portal dark matter models have been examined to account for the Galactic Center gamma-ray excess [13]. It is worth investigating this direction with the general U(1) extension of our scenario with the right-handed neutrino dark matter [28]. 


\section{Acknowledgments}

S.O. would like to thank Ryusuke Endo for encouraging her to carry out the dissertation research. She also wishes to thank Shinsuke Kawai for his valuable advices. S.O. would like to thank the Department of Physics and Astronomy at the University of Alabama for hospitality during her visit for the completion of this work. We would like to record our gratitude to Andy Okada for his encouragements. The work of N.O. is supported in part by the United States Department of Energy grant (de-sc0013680).

\section{References}

[1] P. Minkowski, Phys. Lett. B 67, 421 (1977); T. Yanagida, in Proceedings of the Workshop on the Unified Theory and the Baryon Number in the Universe (O. Sawada and A. Sugamoto, eds.), KEK, Tsukuba, Japan, 1979, p. 95; M. Gell-Mann, P. Ramond, and R. Slansky, Supergravity (P. van Nieuwenhuizen et al. eds.), North Holland, Amsterdam, 1979, p. 315; S. L. Glashow, The future of elementary particle physics, in Proceedings of the 1979 Cargèse Summer Institute on Quarks and Leptons (M. Lévy et al. eds.), Plenum Press, New York, 1980, p. 687; R. N. Mohapatra and G. Senjanović, Phys. Rev. Lett. 44, $912(1980)$.

[2] R. N. Mohapatra and R. E. Marshak, "Local B-L Symmetry Of Electroweak Interactions, Majorana Neutrinos And Neutron Oscillations," Phys. Rev. Lett. 44, 1316 (1980); R. E. Marshak and R. N. Mohapatra, "Quark - Lepton Symmetry And B-L As The U(1) Generator Of The Electroweak Symmetry Group," Phys. Lett. B 91, 222 (1980); C. Wetterich, "Neutrino Masses And The Scale Of B-L Violation," Nucl. Phys. B 187, 343 (1981); A. Masiero, J. F. Nieves and T. Yanagida, "B-L Violating Proton Decay And Late Cosmological Baryon Production," Phys. Lett. B 116, 11 (1982); R. N. Mohapatra and G. Senjanovic, "Spontaneous Breaking Of Global B-L Symmetry And Matter - Antimatter Oscillations In Grand Unified Theories," Phys. Rev. D 27, 254 (1983); W. Buchmuller, C. Greub and P. Minkowski, "Neutrino Masses, Neutral Vector Bosons And The Scale Of B-L Breaking," Phys. Lett. B 267, 395 (1991).

[3] N. Okada and O. Seto, "Higgs portal dark matter in the minimal gauged $U(1)_{B-L}$ model," Phys. Rev. D 82, 023507 (2010) doi:10.1103/PhysRevD.82.023507 arXiv:1002.2525 [hep$\mathrm{ph}]$.

[4] P. H. Frampton, S. L. Glashow and T. Yanagida, "Cosmological sign of neutrino CP violation," Phys. Lett. B 548, 119 (2002) doi:10.1016/S0370-2693(02)02853-8 [hep-ph/0208157]. 
[5] N. Okada and Y. Orikasa, "Dark matter in the classically conformal B-L model," Phys. Rev. D 85, 115006 (2012) doi:10.1103/PhysRevD.85.115006 [arXiv:1202.1405 [hep-ph]]; T. Basak and T. Mondal, "Constraining Minimal $U(1)_{B-L}$ model from Dark Matter Observations," Phys. Rev. D 89, 063527 (2014) doi:10.1103/PhysRevD.89.063527 arXiv:1308.0023 [hep-ph]].

[6] Z. M. Burell and N. Okada, "Supersymmetric minimal B-L model at the TeV scale with right-handed Majorana neutrino dark matter," Phys. Rev. D 85, 055011 (2012) doi:10.1103/PhysRevD.85.055011 [arXiv:1111.1789 [hep-ph]].

[7] S. Khalil and A. Masiero, "Radiative B-L symmetry breaking in supersymmetric models," Phys. Lett. B 665, 374 (2008) doi:10.1016/j.physletb.2008.06.063 [arXiv:0710.3525 [hep$\mathrm{ph}]$.

[8] P. Fileviez Perez and S. Spinner, "The Fate of R-Parity," Phys. Rev. D 83, 035004 (2011) doi:10.1103/PhysRevD.83.035004 arXiv:1005.4930 [hep-ph]].

[9] H. An, R. Huo and L. T. Wang, "Searching for Low Mass Dark Portal at the LHC," Phys. Dark Univ. 2, 50 (2013) doi:10.1016/j.dark.2013.03.002 arXiv:1212.2221 [hep-ph]]; H. An, X. Ji and L. T. Wang, "Light Dark Matter and $Z^{\prime}$ Dark Force at Colliders," JHEP 1207, 182 (2012) doi:10.1007/JHEP07(2012)182 arXiv:1202.2894 [hep-ph]]; D. E. Soper, M. Spannowsky, C. J. Wallace and T. M. P. Tait, "Scattering of Dark Particles with Light Mediators," Phys. Rev. D 90, no. 11, 115005 (2014) doi:10.1103/PhysRevD.90.115005 arXiv:1407.2623 [hep-ph]].

[10] J. Kopp, L. Michaels and J. Smirnov, "Loopy Constraints on Leptophilic Dark Matter and Internal Bremsstrahlung," JCAP 1404, 022 (2014) doi:10.1088/1475-7516/2014/04/022 arXiv:1401.6457 [hep-ph]]; M. Das and S. Mohanty, "Leptophilic dark matter in gauged $L_{\mu}-L_{\tau}$ extension of MSSM," Phys. Rev. D 89, no. 2, 025004 (2014) doi:10.1103/PhysRevD.89.025004 [arXiv:1306.4505 [hep-ph]]; P. Agrawal, Z. Chacko and C. B. Verhaaren, "Leptophilic Dark Matter and the Anomalous Magnetic Moment of the Muon," JHEP 1408, 147 (2014) doi:10.1007/JHEP08(2014)147 [arXiv:1402.7369 [hep-ph]].

[11] L. Basso, O. Fischer and J. J. van der Bij, "Natural Z' model with an inverse seesaw mechanism and leptonic dark matter," Phys. Rev. D 87, no. 3, 035015 (2013) doi:10.1103/PhysRevD.87.035015 arXiv:1207.3250 [hep-ph]]; E. Dudas, L. Heurtier, Y. Mambrini and B. Zaldivar, "Extra U(1), effective operators, anomalies and dark matter," JHEP 1311, 083 (2013) doi:10.1007/JHEP11(2013)083 [arXiv:1307.0005 [hepph]]; X. Chu, Y. Mambrini, J. Quevillon and B. Zaldivar, "Thermal and non-thermal 
production of dark matter via Z'-portal(s)," JCAP 1401, 034 (2014) doi:10.1088/14757516/2014/01/034 [arXiv:1306.4677 [hep-ph]]; E. Ma and R. Srivastava, "Dirac or inverse seesaw neutrino masses with $B-L$ gauge symmetry and $S_{3}$ flavor symmetry," Phys. Lett. B 741, 217 (2015) doi:10.1016/j.physletb.2014.12.049 [arXiv:1411.5042 [hep-ph]]; K. Ghorbani and H. Ghorbani, "Two-portal Dark Matter," Phys. Rev. D 91, no. 12, 123541 (2015) doi:10.1103/PhysRevD.91.123541 [arXiv:1504.03610 [hep-ph]]; E. Ma, N. Pollard, R. Srivastava and M. Zakeri, "Gauge $B-L$ Model with Residual $Z_{3}$ Symmetry," Phys. Lett. B 750, 135 (2015) doi:10.1016/j.physletb.2015.09.010 [arXiv:1507.03943 [hep-ph]].

[12] A. Alves, S. Profumo and F. S. Queiroz, "The dark $Z^{\prime}$ portal: direct, indirect and collider searches," JHEP 1404, 063 (2014) doi:10.1007/JHEP04(2014)063 arXiv:1312.5281 [hepph]]; A. Alves, A. Berlin, S. Profumo and F. S. Queiroz, "Dark Matter Complementarity and the Z' Portal," Phys. Rev. D 92, no. 8, 083004 (2015) doi:10.1103/PhysRevD.92.083004 arXiv:1501.03490 [hep-ph]].

[13] D. Hooper, " $Z$ ' mediated dark matter models for the Galactic Center gamma-ray excess," Phys. Rev. D 91, 035025 (2015) doi:10.1103/PhysRevD.91.035025 arXiv:1411.4079 [hep$\mathrm{ph}]$

[14] The ATLAS collaboration, "Search for new phenomena in the dilepton final state using proton-proton collisions at $\mathrm{s}=13 \mathrm{TeV}$ with the ATLAS detector," ATLAS-CONF-2015070 .

[15] CMS Collaboration [CMS Collaboration], "Search for a Narrow Resonance Produced in $13 \mathrm{TeV}$ pp Collisions Decaying to Electron Pair or Muon Pair Final States," CMS-PASEXO-15-005.

[16] M. Fukugita and T. Yanagida, "Baryogenesis Without Grand Unification," Phys. Lett. B 174, 45 (1986). doi:10.1016/0370-2693(86)91126-3.

[17] S. Iso, N. Okada and Y. Orikasa, "Resonant Leptogenesis in the Minimal B-L Extended Standard Model at TeV," Phys. Rev. D 83, 093011 (2011) doi:10.1103/PhysRevD.83.093011 arXiv:1011.4769 [hep-ph]]; N. Okada, Y. Orikasa and T. Yamada, "Minimal Flavor Violation in the Minimal $U(1)_{B-L}$ Model and Resonant Leptogenesis," Phys. Rev. D 86, 076003 (2012) doi:10.1103/PhysRevD.86.076003 arXiv:1207.1510 [hep-ph]]. 
[18] N. Aghanim et al. [Planck Collaboration], "Planck 2015 results. XI. CMB power spectra, likelihoods, and robustness of parameters," Submitted to: Astron.Astrophys. arXiv:1507.02704 [astro-ph.CO]].

[19] LHC seminar "ATLAS and CMS physics results from Run 2", talks by Jim Olsen and Marumi Kado, CERN, 15 Dec. 2015.

[20] G. Aad et al. [ATLAS Collaboration], "Search for high-mass dilepton resonances in pp collisions at $\sqrt{s}=8 \mathrm{TeV}$ with the ATLAS detector," Phys. Rev. D 90, no. 5, 052005 (2014) doi:10.1103/PhysRevD.90.052005 [arXiv:1405.4123 [hep-ex]].

[21] CMS Collaboration [CMS Collaboration], "Search for Resonances in the Dilepton Mass Distribution in pp Collisions at sqrt(s) = 8 TeV," CMS-PAS-EXO-12-061.

[22] J. Pumplin, D. R. Stump, J. Huston, H. L. Lai, P. Nadolsky and W. K. Tung, "New generation of parton distributions with uncertainties from global QCD analysis," JHEP 07 (2002) 012.

[23] V. D. Barger, W. Y. Keung and E. Ma, "Doubling of Weak Gauge Bosons in an Extension of the Standard Model," Phys. Rev. Lett. 44, 1169 (1980). doi:10.1103/PhysRevLett.44.1169.

[24] M. S. Carena, A. Daleo, B. A. Dobrescu and T. M. P. Tait, “Z' gauge bosons at the Tevatron," Phys. Rev. D 70, 093009 (2004) arXiv:hep-ph/0408098.

[25] S. Schael et al. [ALEPH and DELPHI and L3 and OPAL and LEP Electroweak Collaborations], "Electroweak Measurements in Electron-Positron Collisions at W-BosonPair Energies at LEP," Phys. Rept. 532, 119 (2013) doi:10.1016/j.physrep.2013.07.004 arXiv:1302.3415 [hep-ex]].

[26] J. Heeck, "Unbroken B-L symmetry," Phys. Lett. B 739, $256 \quad$ (2014) doi:10.1016/j.physletb.2014.10.067 [arXiv:1408.6845 [hep-ph]].

[27] T. Appelquist, B. A. Dobrescu and A. R. Hopper, "Nonexotic neutral gauge bosons," Phys. Rev. D 68, 035012 (2003) doi:10.1103/PhysRevD.68.035012 hep-ph/0212073.

[28] N. Okada and S. Okada, work in progress. 